\title{
Optical positions of 68 radio stars
}

\author{
Lu Lizhi $^{1}$, W.T. Manrique ${ }^{2}$ and R. Perdomo ${ }^{3}$ \\ 1 Beijing Astronomical Observatory, Beijing 100080, China \\ 2 Observatorio Astronomico Felix Aguilar, Benavidez 8175, San Juan, Argentina \\ 3 Observatorio Astronomico de La Plata, 1900-La Plata, Argentina
}

Received January 16; accepted January 23, 1996

\begin{abstract}
Optical positions of 68 radio stars observed with the photoelectric astrolabe Mark II of Beijing Astronomical Observatory in San Juan are presented. The Positions of the radio stars are for the epoch of observation and the equinox J2000.0 and a system close that of the system FK5. The mean precisions are $\pm 2.8 \mathrm{~ms}$ and $\pm 0.046^{\prime \prime}$ in right ascensions and declinations, respectively. The magnitudes of stars are from 0.9 mag to 10.7 mag. The declinations are from $-3^{\circ}$ to $-58^{\circ}$. The mean epoch is 1994.2.
\end{abstract}

Key words: stars: general — astronomical data bases: miscellaneous — astrometry — reference system

\section{Introduction}

The photoelectric astrolabe Mark II(PA II) was made at Nanjing Astronomical Instrument Factory in 1974 and was put into operation since Mar. 1976 (Luo 1979). The aperture of object mirror is $200 \mathrm{~mm}$ and the equivalent focal length is $2400 \mathrm{~mm}$. The zenith distance observed is $30^{\circ}$.

Before modernization, the Astrolabe was semiautomatic instrument with the limiting magnitude of 7 mag. In 1988, the automatic observation of the instrument was realized controlled with a micro-computer (PC/AT). And a method of photon-counting was adopted for data processing instead of original electric recorder of time in 1990. The limiting magnitude is increased to more $11 \mathrm{mag}$.

Using the data observed with the instruments from 1976 to 1991, several general catalogues (Zhu et al. 1981; Working Group of GCPA, 1983; Working Group of CGSC, 1991; Lu 1991) of stars had been compiled.

In 1992, the instrument was moved and installed at San Juan Observatory (OAFA) in Argentina for Observations of the catalogue of stars in southern hemisphere.

Radio stars are suitable intermediaries for linking optical stellar reference frames to the quasi-inertial radio reference frame (RRF) represented by compact extragalactic radio sources. From February, 1992 to February, 1995, we observed radio stars with the photoelectric astrolabe using the list provided by H.G. Walter at Astronomishes Rechen Heidelbery (Walter 1990).

This Paper presents the positions of these radio stars for epoch of observation and the equinox J2000.0 and in the system close to that of the FK5.

Send offprint requests to: Lu Lizhi

\section{The observation and reduction}

The observations of radio stars were placed in the reference group of stars.

The residuals of the radio stars are calculated with the corrections of astronomical time, latitude and zenith distance of reference groups of stars. Then, the mean values of the residuals of radio stars are calculated by weighted average.

Assumed $V_{\mathrm{e}}$ and $V_{\mathrm{w}}$ are the residuals reduced to the mean instrumental system after adding the group corrections of reference groups at both eastern and western transits, the position corrections of the radio stars in right ascension and declination from double transits are determined by the formulas:

$$
\Delta \alpha=\frac{V_{\mathrm{e}}-V_{\mathrm{w}}}{30 \cos \varphi_{0}|\sin A|}
$$

and

$$
\Delta \delta=-\frac{V_{\mathrm{e}}+V_{\mathrm{w}}-2 K}{2 \cos q}
$$

where

$\varphi_{0}-$ the adopted value of latitude at the site of the instrument;

$A$ - the azimuth of a star observed, measured eastwards from north;

$q$ - the parallactic angle of a star as it transit the almucantar of the astrolabe.

We used the stars of $|\cos q|<0.2$ (about 490 stars) to calculated $2 K$ from follow:

$$
K=\frac{1}{2}\left(V_{\mathrm{e}}+V_{\mathrm{w}}\right) .
$$


and obtained $2 K=0.020^{\prime \prime} \pm 0.003^{\prime \prime}$.

\section{Results and comparison with CAMC4}

From February, 1992 to February, 1995, there are 68 radio stars to be observed. Using the data observed with the photoelectric astrolabe, the catalogue of radio stars in San Juan (RSSJ) has been compiled from double transits. The mean number of observation of each star is about 80 (38 in the eastern transit, 42 in the western transits) The mean precisions are $\pm 2.8 \mathrm{~ms}$ and $\pm 0.046^{\prime \prime}$ in right ascension and declination, respectively.

There are 42 radio stars in both program between RSSJ and CAMC4 at near observational epoch. So we try to do a comparison between their respective position after reducing to same epoch, 1990. The differences in the sense RSSJ - CAMC4 are shown in the Table 1.

In Table 1, the columns are as follow:

1. Radio stars number of INCA;

2: CAMC4 number;

3: Visual magnitude;

4: Spectral type;

5: Right ascension of RSSJ referred to equinox J2000.0 at epoch 1990;

6: Declination of RSSJ referred to equinox J2000.0 at epoch 1990;

7: Right ascension of CAMC4 referred to equinox J2000.0 at epoch 1990;

8: Declination of CAMC4 referred to equinox J2000.0 at epoch 1990;

9: The difference on right ascension (RSSJ-CAMC4) in unit of $0.001^{\mathrm{s}}$;
10: The difference on declination (RRSJ-CAMC4) in unit of $0.01^{\prime \prime}$;

The catalogue of radio stars observed in San Juan is given in Table 2. The description of each column are:

1. Radio stars number of INCA;

2: SAO number;

3: HD number;

4: Visual magnitude;

5: Spectral type;

6: Right ascension referred to equinox J2000.0 at observation epoch;

8: Mean errors of position on Right ascension in unit of $0.001^{\mathrm{s}}$;

7: Declination referred to equinox J2000.0 at observation epoch;

9: Mean errors of position on declination in unit of $0.01^{\prime \prime}$

10: Number of observations in the eastern transit;

11: Number of observations in the western transit;

12: Mean epoch of observations.

\section{References}

Carlsberg Meridian Catalogue in La Palma, 1989

Lu Lizhi, 1991, Publ. Beijing Astron. Obs. 17, 1

Lu Lizhi, Luo Dingjiang, et al., 1980, Acta Astron. Sin. 22, 305

Luo Dingjiang, et al., 1979, Publ. Beijing Astron. Obs. 1, 56

Walter H.G., et al., 1990, A\&AS 86, 357

Working Group of GCPA, 1983, Acta Astron. Sin. 24, 267

Working Group of CGSC, 1991, Publ. House Surveying and Mapping, Beijing

Zhu Yuanxian, Lu Lizhi, Luo Dingjiang, Ling Zhaofen, 1981, Publ. Beijing Astron. Obs. 1, 1 
Table 1. The difference of positions (RSSJ-CAMC4)

\begin{tabular}{|c|c|c|c|c|c|c|c|c|c|}
\hline \multirow[t]{2}{*}{ INCA } & \multirow[t]{2}{*}{ CAMA4 } & \multirow[t]{2}{*}{ Mag } & \multirow[t]{2}{*}{ Sp } & $\alpha_{\mathrm{rssj}}$ & $\delta_{\mathrm{rssj}}$ & $\alpha_{\text {camc }}$ & $\delta_{\text {camc }}$ & \multirow{2}{*}{$\begin{array}{l}\Delta \alpha \\
\mathrm{ms}\end{array}$} & \multirow{2}{*}{$\begin{array}{c}\Delta \delta \\
0^{\prime \prime} .01\end{array}$} \\
\hline & & & & $\mathrm{m} \mathrm{s}$ & $0{ }^{\prime} \prime \prime$ & $\mathrm{h} \mathrm{m} \mathrm{s}$ & & & \\
\hline 1010 & 200882 & 5.35 & G5 & 11636.353 & -23000.82 & 11636.364 & -23000.73 & -11 & -9 \\
\hline 1043 & 202728 & 8.40 & $\mathrm{~A}$ & 44345.822 & -104056.21 & 44345.816 & -104056.21 & 6 & 0 \\
\hline 1060 & 203132 & 7.00 & G3 & 54039.688 & -201755.79 & 54039.707 & -201755.91 & -19 & 12 \\
\hline 1061 & 203137 & 8.00 & $\mathrm{~A} 0$ & 54102.295 & -24300.85 & $\begin{array}{lll}54 & 02.297\end{array}$ & -24300.82 & -2 & -3 \\
\hline 1075 & 203719 & 6.71 & Wn & 65413.050 & -235541.93 & 65413.046 & -235542.23 & 4 & 30 \\
\hline 1083 & 203955 & 4.90 & M2 & 73347.968 & -143126.08 & 73347.962 & -143126.03 & 6 & -5 \\
\hline 1092 & 204385 & 7.20 & G2 & 85942.760 & 0000.00 & 85942.742 & -274858.28 & 18 & \\
\hline 1093 & 306733 & 7.45 & K0 & 92449.040 & -234934.61 & 92449.048 & -234934.30 & -8 & -31 \\
\hline 1097 & 406021 & 8.84 & $\mathrm{~K} 2$ & 93713.024 & & 93712.998 & -420114.70 & 26 & \\
\hline 1116 & 308446 & 8.10 & G5 & 113922.239 & & 113922.244 & -392307.21 & -5 & \\
\hline 1155 & 310689 & 9.80 & G5 & 143548.437 & -180211.25 & 143548.426 & -180211.49 & 11 & 24 \\
\hline 1190 & 206894 & 5.97 & O5 & 180352.450 & -242138.63 & 180352.441 & -242138.61 & 9 & -2 \\
\hline 1198 & 207024 & 9.70 & O9 & 182531.469 & -124124.38 & 182531.492 & -124124.25 & -23 & -13 \\
\hline 1208 & 315232 & 6.90 & K0 & 192240.299 & -203833.47 & 192240.298 & -203833.80 & 1 & 33 \\
\hline 1209 & 207559 & 9.20 & G5 & 192257.224 & -141531.78 & 192257.212 & -141531.99 & 12 & 21 \\
\hline 1210 & 413340 & 9.60 & K0 & 192805.543 & & 192805.505 & -405005.15 & 38 & \\
\hline 1212 & 207751 & 8.30 & G5 & 193938.804 & -60349.29 & 193938.799 & -60349.08 & 5 & -21 \\
\hline 1226 & 316144 & 9.00 & K0 & 202936.859 & -210734.70 & 202936.862 & -210734.40 & -3 & -30 \\
\hline 1238 & 208993 & 7.80 & K0 & $21 \quad 1452.750$ & & 211452.752 & -311100.58 & -2 & \\
\hline 1242 & 317194 & 10.77 & G5 & 213948.889 & -160021.09 & 213948.872 & -160020.99 & 17 & -10 \\
\hline 2003 & 200228 & 6.39 & G2 & 02251.515 & -121234.52 & 02251.505 & -121235.04 & 10 & 52 \\
\hline 2083 & 202464 & 7.10 & G2 & 40940.870 & -75335.30 & 40940.868 & -75335.42 & 2 & 12 \\
\hline 2115 & 304046 & 6.54 & B2 & 53521.883 & -42939.06 & 53521.858 & -42939.13 & 25 & 7 \\
\hline 2167 & 305357 & 5.79 & G8 & 72751.683 & -113325.18 & 72751.664 & -113324.83 & 19 & -35 \\
\hline 2290 & 410516 & 7.40 & K0 & 152326.070 & -63636.59 & 152326.059 & -63636.51 & 11 & -8 \\
\hline 2470 & 104462 & 8.00 & G5 & 213416.560 & -132901.58 & 213416.564 & -132901.50 & -4 & -8 \\
\hline 2602 & 302705 & 8.29 & $\mathrm{~A} 4$ & 33225.145 & $-3 \quad 1847.77$ & 33225.148 & -31847.68 & -3 & -9 \\
\hline 2603 & 305266 & 5.70 & F1 & 71928.067 & -162341.68 & 71928.066 & -162341.66 & 1 & -2 \\
\hline 2605 & 308764 & 4.02 & $\mathrm{~F} 2$ & 120824.752 & -244343.61 & 120824.728 & -244343.62 & 24 & 1 \\
\hline 2607 & 408760 & 8.98 & $\mathrm{~A} 2$ & 131520.783 & -172816.82 & 131520.756 & -172817.13 & 27 & 31 \\
\hline 2610 & 311909 & 8.20 & F5 & 155427.056 & & 155427.052 & -302531.00 & 4 & \\
\hline 2652 & 301370 & 8.10 & G5 & 14641.538 & -240051.26 & 14641.539 & -240051.41 & -1 & 15 \\
\hline 2653 & 201760 & 8.10 & G5 & 24325.506 & & 24325.476 & -375541.93 & 30 & \\
\hline 2657 & 402781 & 7.10 & K0 & 51430.531 & -261230.79 & 51430.520 & -261230.54 & 11 & -25 \\
\hline 2659 & 404542 & 7.00 & K0 & 73613.773 & & 73613.774 & -445727.01 & -1 & \\
\hline 2660 & 405128 & 7.80 & G5 & 82514.155 & $\begin{array}{lll}-7 & 10 & 12.79\end{array}$ & 82514.165 & -7 1013.00 & -10 & 21 \\
\hline 2661 & 405211 & 6.36 & K0 & 83258.531 & & 83258.521 & -343802.37 & 10 & \\
\hline 2663 & 406942 & 8.20 & $\mathrm{~K} 2$ & 105314.896 & & 105314.880 & -325918.95 & 16 & \\
\hline 2664 & 407969 & 8.30 & K0 & 121320.708 & -9 0447.07 & 121320.703 & -9 0447.04 & 5 & -3 \\
\hline 2665 & 309814 & 8.84 & $\mathrm{~K} 2$ & 133608.312 & & 133608.310 & -332844.54 & 2 & \\
\hline 2666 & 206583 & 6.70 & K2 & 171613.728 & & 171613.722 & -263234.91 & 6 & \\
\hline 2710 & 206416 & 8.10 & K5 & 165417.937 & -64242.60 & 165417.917 & -64242.43 & 20 & -17 \\
\hline
\end{tabular}


Table 2. Optical positions of radio stars observed in San Juan

\begin{tabular}{|c|c|c|c|c|c|c|c|c|c|c|c|}
\hline \multirow[t]{2}{*}{ INCA } & \multirow[t]{2}{*}{$\mathrm{SAO}$} & \multirow[t]{2}{*}{ HD } & \multirow[t]{2}{*}{ Mag } & \multirow[t]{2}{*}{$\mathrm{Sp}$} & $\alpha_{2000}$ & \multirow{2}{*}{$\begin{array}{c}M_{\alpha} \\
\mathrm{ms}\end{array}$} & $\delta_{2000}$ & \multirow{2}{*}{$\begin{array}{c}M_{\delta} \\
0^{\prime \prime} .01\end{array}$} & \multirow[t]{2}{*}{$N_{\mathrm{e}}$} & \multirow[t]{2}{*}{$N_{\mathrm{w}}$} & \multirow{2}{*}{$\begin{array}{l}\text { Epoch } \\
1900+\end{array}$} \\
\hline & & & & & $\mathrm{h} \mathrm{m} \quad \mathrm{s}$ & & & & & & \\
\hline 1010 & 7672 & 129204 & 5.35 & G5 & 11636.326 & 8.1 & -23001.10 & 3.0 & 22 & 30 & 94.26 \\
\hline 1032 & 26354 & 233401 & 9.30 & K1 & 40729.119 & 2.7 & -523416.00 & 3.8 & 33 & 69 & 94.86 \\
\hline 1043 & 30050 & 149847 & 8.40 & $\mathrm{~A}$ & 44345.825 & 3.1 & -104056.15 & 4.2 & 37 & 15 & 94.53 \\
\hline 1060 & 37847 & 170678 & 7.00 & G3 & 54039.696 & 2.2 & -201755.84 & 5.7 & 47 & 47 & 93.97 \\
\hline 1061 & 37806 & 132452 & 8.00 & A0 & 54102.301 & 4.7 & -24300.87 & 1.9 & 17 & 38 & 94.97 \\
\hline 1062 & 39937 & 234181 & 7.30 & F7 & 55220.195 & 4.2 & -570921.83 & 3.0 & 33 & 37 & 94.02 \\
\hline 1075 & 50896 & 172546 & 6.71 & Wn & 65413.052 & 1.8 & -235541.93 & 6.2 & 30 & 55 & 94.03 \\
\hline 1076 & 51268 & 234770 & 9.10 & $\mathrm{~K} 2$ & 65333.575 & 3.8 & -545259.03 & 3.9 & 30 & 36 & 94.53 \\
\hline 1078 & 54791 & 218478 & 9.90 & G8 & 70821.046 & 2.0 & & & 50 & 36 & 94.89 \\
\hline 1079 & 56096 & 218549 & 2.60 & M5 & 71332.261 & 2.1 & & & 38 & 46 & 94.27 \\
\hline 1083 & 60414 & 153072 & 4.90 & M2 & 73347.967 & 2.1 & -143126.07 & 3.8 & 31 & 40 & 94.34 \\
\hline 1087 & 66811 & 198752 & 2.25 & O5 & 80335.065 & 2.0 & & & 45 & 44 & 94.82 \\
\hline 1092 & 77137 & 176805 & 7.20 & G2 & 85942.748 & 1.8 & & & 44 & 18 & 93.97 \\
\hline 1093 & 81410 & 177412 & 7.45 & K0 & 92449.033 & 1.7 & -234934.71 & 5.6 & 44 & 52 & 94.03 \\
\hline 1097 & 83442 & 221347 & 8.84 & $\mathrm{~K} 2$ & 93712.997 & 1.9 & & & 37 & 38 & 93.88 \\
\hline 1112 & 96751 & & 9.80 & G3 & 110756.354 & 3.0 & $\begin{array}{lll}-51 & 07 & 07.92\end{array}$ & 5.1 & 47 & 52 & 94.44 \\
\hline 1114 & 98803 & 222744 & 9.00 & G6 & 112144.513 & 2.5 & -495407.75 & 5.1 & 33 & 56 & 94.57 \\
\hline 1116 & 101309 & 202671 & 8.10 & G5 & 113922.240 & 1.8 & & & 85 & 26 & 93.20 \\
\hline 1123 & 103197 & & 9.90 & K1 & 115253.016 & 3.2 & -501734.03 & 6.1 & 61 & 31 & 94.47 \\
\hline 1124 & 103855 & 223131 & 9.20 & G8 & 115726.725 & 2.7 & -483930.79 & 6.4 & 41 & 35 & 94.04 \\
\hline 1141 & 117600 & 224225 & 9.90 & $\mathrm{~K} 2$ & 133216.213 & 3.0 & -472506.40 & 8.4 & 23 & 90 & 94.19 \\
\hline 1155 & 128171 & 158665 & 9.80 & G5 & 143548.431 & 2.6 & -180211.33 & 5.9 & 30 & 53 & 94.38 \\
\hline 1168 & 139084 & 242791 & 8.10 & G5 & 153857.595 & 7.3 & -574226.72 & 4.6 & 16 & 13 & 94.30 \\
\hline 1173 & 146550 & & 10.10 & F6 & 161841.509 & 3.0 & & & 40 & 31 & 93.99 \\
\hline 1176 & 148478 & 184415 & .90 & M1 & 162924.459 & 1.9 & & & 22 & 41 & 94.45 \\
\hline 1182 & & & 9.80 & pe & 170901.019 & 7.5 & -565447.83 & 5.5 & 11 & 26 & 94.23 \\
\hline 1190 & 164794 & 186204 & 5.97 & O5 & 180352.452 & 3.0 & -242138.64 & 10.6 & 18 & 43 & 93.03 \\
\hline 1198 & 169515 & 161458 & 9.70 & O9 & 182531.471 & 3.5 & -124124.39 & 5.6 & 19 & 27 & 94.54 \\
\hline 1202 & 174429 & 245781 & 8.36 & K0 & 185305.860 & 3.2 & -501049.29 & 6.2 & 24 & 45 & 93.74 \\
\hline 1208 & 181809 & 188043 & 6.90 & K0 & 192240.301 & 1.9 & -203833.87 & 5.0 & 53 & 57 & 93.90 \\
\hline 1209 & 181943 & 162546 & 9.20 & G5 & 192257.240 & 2.7 & -141531.81 & 4.7 & 32 & 47 & 94.08 \\
\hline 1210 & 182776 & 229695 & 9.60 & K0 & 192805.555 & 1.7 & & & 114 & 38 & 93.82 \\
\hline 1212 & 185510 & 143657 & 8.30 & G5 & 193938.812 & 3.4 & -60349.39 & 2.9 & 35 & 42 & 93.70 \\
\hline 1226 & 195040 & 189349 & 9.00 & K0 & 202936.862 & 2.5 & -210734.73 & 7.0 & 19 & 43 & 94.43 \\
\hline 1238 & 202134 & 212824 & 7.80 & K0 & 211452.731 & 2.4 & & & 25 & 43 & 94.60 \\
\hline 1242 & 206046 & 164558 & 10.77 & G5 & 213948.905 & 2.7 & -160021.09 & 5.3 & 32 & 28 & 94.61 \\
\hline 1254 & 214479 & 191294 & 9.07 & M1 & 223845.403 & 2.8 & -203715.27 & 7.6 & 22 & 26 & 94.42 \\
\hline 2003 & 1835 & 147237 & 6.39 & G2 & 02251.635 & 2.2 & -121234.26 & 3.4 & 40 & 53 & 94.45 \\
\hline 2083 & 26337 & 130994 & 7.10 & G2 & 40940.889 & 2.4 & -75334.86 & 2.5 & 42 & 40 & 94.24 \\
\hline 2115 & 37017 & 132317 & 6.54 & $\mathrm{~B} 2$ & 53521.885 & 4.7 & -42939.05 & 3.2 & 25 & 29 & 94.16 \\
\hline 2167 & 59067 & 152909 & 5.79 & G8 & 72751.683 & 2.0 & -113325.17 & 2.9 & 61 & 42 & 93.98 \\
\hline 2290 & 136905 & 140499 & 7.40 & K0 & 152326.066 & 3.1 & -63637.04 & 2.8 & 37 & 35 & 93.98 \\
\hline 2470 & 205249 & 164484 & 8.00 & G5 & 213416.565 & 1.7 & -132901.56 & 2.9 & 97 & 68 & 94.01 \\
\hline 2476 & 207098 & 164644 & 2.81 & A7 & 214702.334 & 2.0 & -160736.51 & 4.0 & 54 & 45 & 93.82 \\
\hline 2602 & 21985 & 130554 & 8.29 & $\mathrm{~A} 4$ & 33225.136 & 5.9 & -31847.93 & 3.0 & 21 & 45 & 94.16 \\
\hline
\end{tabular}


Table 2. continued

\begin{tabular}{|c|c|c|c|c|c|c|c|c|c|c|c|}
\hline \multirow[t]{2}{*}{ INCA } & \multirow[t]{2}{*}{$\mathrm{SAO}$} & \multirow[t]{2}{*}{ HD } & \multirow[t]{2}{*}{ Mag } & \multirow[t]{2}{*}{$\mathrm{Sp}$} & $\alpha_{2000}$ & \multirow{2}{*}{$\begin{array}{c}M_{\alpha} \\
\mathrm{ms}\end{array}$} & $\delta_{2000}$ & \multirow{2}{*}{$\begin{array}{c}M_{\delta} \\
0^{\prime \prime} .01\end{array}$} & \multirow[t]{2}{*}{$N_{\mathrm{e}}$} & \multirow[t]{2}{*}{$N_{\mathrm{w}}$} & \multirow{2}{*}{$\begin{array}{l}\text { Epoch } \\
1900+\end{array}$} \\
\hline & & & & & $\mathrm{h} \quad \mathrm{m} \quad \mathrm{s}$ & & & & & & \\
\hline 2603 & 57167 & 152724 & 5.70 & F1 & 71928.120 & 1.7 & -162342.25 & 3.5 & 37 & 96 & 94.27 \\
\hline 2605 & 105452 & 180505 & 4.02 & $\mathrm{~F} 2$ & 120824.781 & 1.9 & -244343.78 & 6.7 & 62 & 17 & 94.37 \\
\hline 2607 & 115122 & 157818 & 8.98 & $\mathrm{~A} 2$ & 131520.761 & 2.4 & -172816.93 & 5.3 & 17 & 55 & 93.35 \\
\hline 2609 & 132742 & 140270 & 4.91 & $\mathrm{~A} 0$ & 150058.382 & 2.3 & -83108.25 & 2.6 & 33 & 53 & 93.53 \\
\hline 2610 & 142217 & 207103 & 8.20 & F5 & 155427.053 & 1.6 & & & 62 & 61 & 94.06 \\
\hline 2652 & 10909 & 167287 & 8.10 & G5 & 14641.584 & 1.8 & -240050.84 & 6.2 & 40 & 33 & 94.27 \\
\hline 2653 & 17084 & 193879 & 8.10 & G5 & 24325.534 & 1.7 & & & 57 & 58 & 93.94 \\
\hline 2657 & 34198 & 170230 & 7.10 & K0 & 51430.548 & 1.7 & -261230.99 & 7.1 & 46 & 53 & 94.22 \\
\hline 2659 & 61245 & 218831 & 7.00 & K0 & 73613.788 & 2.3 & & & 39 & 46 & 94.03 \\
\hline 2660 & 71071 & 135893 & 7.80 & G5 & 82514.124 & 2.8 & -7 1012.90 & 2.7 & 35 & 44 & 94.49 \\
\hline 2661 & 72688 & 199353 & 6.36 & K0 & 83258.525 & 2.2 & & & 30 & 47 & 94.83 \\
\hline 2663 & 94389 & 201857 & 8.20 & $\mathrm{~K} 2$ & 105314.888 & 1.5 & & & 36 & 39 & 94.26 \\
\hline 2664 & 106225 & 138652 & 8.30 & K0 & 121320.701 & 2.4 & -9 0447.01 & 2.9 & 55 & 36 & 94.47 \\
\hline 2665 & 118238 & 204640 & 8.84 & $\mathrm{~K} 2$ & 133608.309 & 2.5 & & & 33 & 21 & 94.29 \\
\hline 2666 & 156026 & 185213 & 6.70 & $\mathrm{~K} 2$ & 171613.596 & 2.2 & & & 25 & 31 & 93.71 \\
\hline 2701 & 6882 & 232306 & 3.91 & F7 & 10823.071 & 3.5 & -551444.99 & 3.4 & 44 & 40 & 94.76 \\
\hline 2703 & 19754 & 130323 & 7.80 & K0 & 31038.497 & 3.7 & -52338.09 & 2.9 & 16 & 46 & 94.39 \\
\hline 2704 & 39576 & 170952 & 9.50 & G0 & 55215.988 & 2.4 & & & 32 & 32 & 94.61 \\
\hline 2707 & 82558 & 155272 & 7.50 & K0 & 93225.676 & 2.4 & $-11 \quad 1104.96$ & 3.4 & 34 & 19 & 94.16 \\
\hline 2709 & 123485 & 224722 & 8.90 & A0 & 140902.190 & 2.9 & & & 31 & 34 & 93.79 \\
\hline 2710 & 152556 & 141428 & 8.10 & K5 & 165417.936 & 3.6 & -64242.61 & 3.4 & 30 & 37 & 93.98 \\
\hline 2711 & 161741 & 209291 & 8.20 & B9 & 174847.622 & 2.0 & & & 58 & 35 & 94.08 \\
\hline 2715 & 187949 & 163080 & 6.46 & $\mathrm{~A} 2$ & 195306.385 & 1.9 & -143611.22 & 3.3 & 38 & 60 & 94.38 \\
\hline
\end{tabular}

